\title{
A Comparative Study Of Butorphanol As An Adjunct To Bupivacaine In Comparison To Bupivacaine Alone.
}

\author{
Prof.Dr.Subrata Nag,Dr.Ritesh kumar,Dr.Shakir najfi,Dr.Mahima Lakhanpal
}

\begin{abstract}
Neuraxial opioids are widely used in conjunction with local anaesthetics (LA) as they permit the use of lower dose of LA while providing adequate anaesthesia and analgesia. Neuraxial opioids also allow prolonged analgesia in the postoperative period and faster recovery from spinal anaesthesia. Antinociceptive synergism between LA and intrathecal opioids has been demonstrated in various animal studies. The present study was undertaken to compare the safety and efficacy of anaesthesia and hemodynamic stability of different doses of intrathecal bupivacaine-butorphanol mixture with intrathecal bupivacaine alone for lower abdominal \& lower limb surgery. This study was conducted as only a limited number of studies have explored the use of intrathecal butorphanol in human subjects previously. Butorphanol is a lipophilic opioid agonist-antagonist analgesic with a published affinity for opioid receptors in vitro of 1:4:25 (mu: delta: kappa). Studies have reported a dose-dependent increase in the duration of analgesia provided by epidural butorphanol for relief of post-caesarean section pain.
\end{abstract}

\section{Methods}

Fourty six patients of ASA grade I and II between 18-58yrs of age of either sex, scheduled for elective lower abdominal \& lower limb surgical procedure were included The study protocol was approved by Institutional Ethical Committee and written informed consent was obtained from all patients.

The pre-anaesthetic check-up included a detailed medical and surgical history, and any previous anaesthetic exposure with its outcome. General examination includes general condition, built, weight, pulse rate, blood pressure, respiratory rate, and presence of cyanosis, anaemia, clubbing, jaundice or edema. A careful thorough systemic examination was done to rule out any cardiovascular, respiratory, gastrointestinal and neurological or any other systemic illness.Routine biochemistry investigation included haemoglobin, total leucocyte count, differential leucocyte count, blood sugar, blood urea, and serum creatinine, were done in all patients. ECG and X-Ray Chest were done in patients where indicated and in those over 40 years of age along with other relevant investigation.

After taking detailed history and thorough clinical examination, the patients were excluded from the study on the basis of below mentioned criteria:

Patients with systemic hypertension, hepatic dysfunction, renal dysfunction, endocrine dysfunction, cardiac dysfunction, morbid obesity (body weight more than $20 \%$ of the ideal body weight), Other exclusion criteria were patients with known drug hypersensitivity, those on antihypertensive medication or antidepressant drugs and those who refused to give consent.

Patients using2-adrenergic receptors antagonists, calcium channel blockers, angiotensin converting enzyme inhibitors, ornoted to have dysrhythmias on the electrocardiogram(ECG), a body weight of more than $120 \mathrm{~kg}$, or height less than $150 \mathrm{~cm}$ were excluded from the study. Standardmonitoring was used, including non-invasive arterial blood pressure, ECG, heart rate (HR) and pulseoximetry (Spo2).

The total 60 patients were randomly divided into two groups of 30 patients each according to a computer generated random table. Group A $(\mathrm{n}=30)$ patients received $15 \mathrm{mg}$ of bupivacaine \& $1 \mathrm{ml}$ of NS and Group D $(\mathrm{n}=30)$ patients received bupivacane $15 \mathrm{mg} \& .05 \mathrm{mg}$. Group allocation was done by an assistant who was unaware of the study protocol and was not involved in the study.

\section{Anaesthetic technique}

Patients were premedicated with tab. alprazolam $0.25 \mathrm{mg}$ and tab. Ranitidine $150 \mathrm{mg}$ the night before the surgery. All patients were kept fasting for 8 hours prior to surgery.

On arrival to operation theatre routine monitoring was started and base line vital parameters of heart rate, systemic arterial pressure including systolic, diastolic and mean arterial pressure, arterial oxygen saturation (SpO2), and ECG were recorded. An intravenous line was secured and Ringer lactate was given at rate of 6-8 $\mathrm{ml} / \mathrm{kg}$. All patients received premedication of intravenous Inj. Midazolam $(0.02 \mathrm{mg} / \mathrm{kg})$ and inj. glycopyrrolate $(0.01 \mathrm{mg} / \mathrm{kg})$..

After preparation with the patient in sitting position spinal anaesthesia was performed at L3-L4 level through a midline approach with quincke needle.Study group A patients were given bupivacaine $15 \mathrm{mg} \& 1 \mathrm{ml}$ of NS (total volume $4 \mathrm{ml}$ ) and group B bupivacaine $15 \mathrm{mg} \& 0.5 \mathrm{mg}$ butorphenol(total volume $4 \mathrm{ml}$ ). Study 
medication was prepared by an anaesthesiologist who was blinded to the randomization schedule.the anaesthesiologist performing block recorded baseline vitals preoperatively,every 3 min for first 15 min,then every 5 min until patientdischarged from PACU.sensory dermatome was assessed by pinprick \& motor by modified bromage score

Results

Out of 60 patients three patient from group A \& two from group B were considered as failure.55 patients completed study protocol and included in data.the onset of block $\&$ duration is given in Table 1.The various vitals monitoring before during and after procedure are arranged in table 2.

Table 1.

Gender

$$
\text { Group A(n=27) Group D(n=28) }
$$

Male

Female

Surgery

Abdominal \& vaginal

hystrectomy

8

Appendicitis

Hernia

Turp

Skin grafting

5

7

3

4
7

8

6

4

3

\begin{tabular}{|l|l|l|l|}
\hline & Group A $(\mathbf{n = 2 7})$ & Group B $(\mathbf{n = 2 8})$ & p value \\
\hline $\begin{array}{l}\text { Age } \\
(\mathbf{y r s})\end{array}$ & $32.54 \pm 10.73$ & $34.07 \pm 12.03$ & 0.39 \\
\hline $\begin{array}{l}\text { Sex } \\
(\mathrm{M} / \mathrm{F})\end{array}$ & $14 / 13$ & $14 / 14$ & 0.273 \\
\hline $\mathrm{Ht}(\mathrm{cm})$ & $149.73 \pm 4.76$ & $149.03 \pm 5.77$ & 0.61 \\
\hline $\mathrm{Wt}(\mathrm{Kg})$ & $55.8 \pm 8.41$ & $53.6 \pm 9.44$ & 0.28 \\
\hline $\begin{array}{l}\text { ASA } \\
\text { grade }\end{array}$ & $20 / 7$ & $21 / 6$ & 0.198 \\
\hline I/II & & & \\
\hline
\end{tabular}

$\underline{\text { Various surgical interventions done in patients in both groups }}$

\begin{tabular}{|l|l|} 
Surgery & Group A
\end{tabular}

\section{Group B}

8

6

7

3

4 
Comparative evaluation of Mean Systolic Blood Pressure (mm Hg)

\begin{tabular}{|r|c|c|c|}
\hline $\begin{array}{c}\text { Time } \\
\text { interval in min }\end{array}$ & Group A & Group B & Pvalue \\
\hline Base & $137.19 \pm 13.18$ & $134.79 \pm 14.62$ & 0.14 \\
\hline $\begin{array}{l}3 \text { min after } \\
\text { spinal } \\
\text { anesthesia }\end{array}$ & $131.06 \pm 13.09$ & $130.56 \pm 13.07$ & 0.40 \\
\hline 6 min & $127.80 \pm 13.74$ & $126.34 \pm 12.25$ & 0.33 \\
\hline 12 min & $125.98 \pm 15.79$ & $124.66 \pm 19.44$ & 0.22 \\
\hline
\end{tabular}

\begin{tabular}{|r|c|c|c|}
\hline $15 \mathrm{~min}$ & $119.32 \pm 15.72$ & $118.92 \pm 12.60$ & 0.09 \\
\hline $30 \mathrm{~min}$ & $116.21 \pm 12.40$ & $116.43 \pm 12.20$ & 0.40 \\
\hline $45 \mathrm{~min}$ & $115.68 \pm 10.94$ & $116.10 \pm 10.45$ & 0.16 \\
\hline $60 \mathrm{~min}$ & $112.80 \pm 10.40$ & $115.16 \pm 10.70$ & 0.40 \\
\hline $75 \mathrm{~min}$ & $112.78 \pm 10.01$ & $114.39 \pm 10.35$ & 0.30 \\
\hline erative & $113.65 \pm 7.71$ & $113.34 \pm 8.14$ & 0.45 \\
\hline
\end{tabular}

\section{Comparative evaluation of Mean Diastolic Blood Pressure (mm Hg)}

\begin{tabular}{|c|c|c|c|}
\hline Time interval in min & Group A & Group B & P-value \\
\hline Base line & $85.85 \pm 9.07$ & $86.08 \pm 9.99$ & 0.43 \\
\hline $\begin{array}{l}3 \text { min after spinal } \\
\text { anesthesia }\end{array}$ & $82.59 \pm 7.86$ & $79.61 \pm 9.40$ & $0.01^{*}$ \\
\hline 6 min & $80.93 \pm 7.78$ & $77.26 \pm 10.33$ & $0.006^{* *}$ \\
\hline 9 min & $80.21 \pm 8.18$ & $75.38 \pm 9.68$ & $0.0005^{* *}$ \\
\hline 12 min & $78.52 \pm 8.21$ & $73.61 \pm 9.15$ & $0.0003^{* *}$ \\
\hline 15 min & $75.69 \pm 10.89$ & $73.28 \pm 8.38$ & $0.04 *$ \\
\hline
\end{tabular}


A Comparative Study Of Butorphanol As An Adjunct To Bupivacaine In Comparison

\begin{tabular}{|c|c|c|c|}
\hline $30 \mathrm{~min}$ & $74.57 \pm 8.29$ & $72.43 \pm 8.47$ & $0.05^{*}$ \\
\hline $45 \mathrm{~min}$ & $74.52 \pm 8.59$ & $71.33 \pm 8.32$ & $0.01^{*}$ \\
\hline $60 \mathrm{~min}$ & $73.44 \pm 7.59$ & $70.53 \pm 6.98$ & $0.007 * *$ \\
\hline 75 min & $72.88 \pm 6.95$ & $72.67 \pm 6.96$ & 0.42 \\
\hline 90 min & $71.86 \pm 6.77$ & $73.05 \pm 6.37$ & 0.13 \\
\hline Postoperative & $72.48 \pm 6.52$ & $73.64 \pm 6.47$ & 0.13 \\
\hline
\end{tabular}

\section{Comparative evaluation of Mean Heart rate (beats/min)}

\begin{tabular}{|c|c|c|c|}
\hline $\begin{array}{l}\text { Time interval in } \\
\text { min }\end{array}$ & Group A & Group B & $P$-value \\
\hline Base line & $100.55 \pm 11.47$ & $88.03 \pm 11.57$ & 1.0 \\
\hline $\begin{array}{l}1 \text { min after spinal } \\
\text { anesthesia }\end{array}$ & $97.98 \pm 10.05$ & $88.08 \pm 11.06$ & 1.0 \\
\hline $3 \mathrm{~min}$ & $96.60 \pm 9.17$ & $82.87 \pm 11.74$ & 0.17 \\
\hline $5 \mathrm{~min}$ & $95.06 \pm 10.02$ & $74.67 \pm 10.51$ & $0.0 * *$ \\
\hline $10 \mathrm{~min}$ & $92.68 \pm 9.53$ & $72.29 \pm 10.69$ & $0.0 * *$ \\
\hline $15 \mathrm{~min}$ & $90.23 \pm 12.08$ & $69.21 \pm 9.41$ & $0.0 * *$ \\
\hline $30 \mathrm{~min}$ & $87.68 \pm 8.91$ & $68.61 \pm 10.02$ & $0.0 * *$ \\
\hline $45 \mathrm{~min}$ & $86.98 \pm 8.79$ & $66.70 \pm 9.09$ & $0.0 * *$ \\
\hline $60 \mathrm{~min}$ & $85.48 \pm 8.30$ & $65.92 \pm 8.06$ & $0.0 * *$ \\
\hline 75 min & $86 \pm 8.59$ & $64.91 \pm 7.41$ & $0.0 * *$ \\
\hline $90 \mathrm{~min}$ & $84.05 \pm 6.07$ & $65.34 \pm 7.06$ & $0.0 * *$ \\
\hline Postoperative & $83.67 \pm 5.44$ & $66.38 \pm 5.94$ & $0.0 * *$ \\
\hline
\end{tabular}




\section{$\underline{\text { Intra-operative and postoperative adverse events }}$}

\begin{tabular}{|l|l|l|}
\hline & Group A & Group B \\
\hline $\begin{array}{l}\text { Adverse } \\
\text { Events }\end{array}$ & No. of patient & No. of patient \\
\hline Hypotension & 2 & 4 \\
\hline Bradycardia & 1 & 4 \\
\hline Shivering & 1 & 5 \\
\hline Headache & 0 & 0 \\
\hline Nausea & 0 & 2 \\
\hline
\end{tabular}

\section{Conclusion}

- Butorphenol when used as an adjunct to bupivacaine in spinal anaesthesia helped in keeping the patient hemodynamically stable throughout the surgery in comparison to bupivacaine alone.

- Although no major neurological complications have been reported so far, larger studies are required to rule out any short term or long term adverse effects.

\section{References}

[1] 1.A comparative evaluation of intrathecal bupivacaine alone, sufentanil or butorphanol in combination with bupivacaine for endoscopic urological surgery

[2] Manpreet Kaur, Sunil Katyal, ${ }^{1}$ Suneet Kathuria, ${ }^{1}$ and Prabhjot Singh

[3] Saudi J Anaesth. 2011 Apr-Jun; 5(2): 202-207

[4] 2.Comparison of intrathecal bupivacaine-fentanyl and bupivacaine-butorphanol mixtures for lower limb orthopedic procedures Binay Kumar, Aparna Williams, Dootika Liddle, Mary Department of Anesthesia and Critical Care, Christian Medical College and Hospital, Ludhiana, Punjab, India

[5] 3.Side effects of intrathecal and epidural opioids

[6] Mark A. Chaney ,Canadian Journal of Anaesthesia ,October 1995, Volume 42, Issue 10, pp 891-903

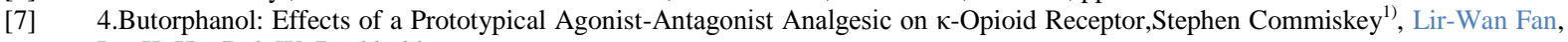
Ing K. Ho, Rob W. Rockhold

[8] 5.Department of Pharmacology \& Toxicology, University of Mississippi Medical Center Released 2005/06/18 [Advance Publication] Released 2005/06/08

[9] 6.Supersensitivity of spinal opioid receptors to antagonists in intrathecal butorphanol and morphine dependence Wichai Wongchanapai ${ }^{\mathrm{a}}$, Brian K. Tsang ${ }^{\mathrm{b}}$, Zhi He $\mathrm{b}^{\mathrm{b}}$, Tangeng Ma ${ }^{\mathrm{a}}$, Ing K. Ho ${ }^{\mathrm{a}}$ Department of Pharmacology and Toxicology, University of Mississippi Medical Center, Jackson, Mississippi 39216, USA, Department of Anesthesiology, University of Mississippi Medical Center, Jackson, Mississippi 39216, USA 